real science more compelling.

Percival's Planet ends before Tombaugh's death, a graceful effort to avoid Pluto's miserable - albeit scientifically justified demotion. But there is a neat coda to the tale.
Some of Tombaugh's ashes are now on board the New Horizons mission to that distant, frigid dwarf planet. Speeding through the outer planets, his ashes will arrive in 2015 at the speck caught by his sharp eye a lifetime ago.
Not bad for a farmer's boy from Kansas. Caleb Scharf is director of astrobiology at Columbia University, New York 10027, USA, and author of Extrasolar Planets and Astrobiology. e-mail: caleb@astro.columbia.edu

\title{
Behind the Mona Lisa's smile
}

\section{X-ray scans reveal Leonardo's remarkable control of glaze thickness, explains Philip Ball.}

Leonardo da Vinci was renowned as a prevaricating genius, apt to undertake too much, to experiment open-endedly and to stall over details. "This man will never do anything!", Pope Leo X is said to have complained after finding the artist concocting a new kind of varnish rather than beginning a commissioned work. Even Leonardo's Mona Lisa portrait was never formally completed, although he laboured on it for four years beginning in 1503 , and returned to it many times throughout his life.

A study of the Mona Lisa's paint layers, published in Angewandte Chemie International Edition last month (L. de Viguerie et al. Angew. Chem. Int. Ed. doi:10.1002/anie.201001116; 2010), gives insight into the techniques over which Leonardo obsessed. Philippe Walter and his colleagues at the Centre for Research and Restoration of French Museums - based, like the painting, in the Louvre in Paris - found that the smooth shading of the iconic face is a product of astonishingly fine control of glaze thickness, and that Leonardo experimented widely with painting methods and materials on other portraits.

Rather than extract paint samples from the sacrosanct flesh tones of the Mona Lisa's face, Walter and colleagues exploited a non-invasive technique that has only recently been applied to art analysis: X-ray fluorescence spectroscopy. Bombardment of the material with $\mathrm{X}$-rays excites an electronic transition from an atom's inner shell. The excited electron then decays by emitting another X-ray, the energy of which reveals the atom's elemental identity. Thanks to improvements in instrumentation and in software - developed when the team worked with other artworks using the bright $\mathrm{X}$-ray source at the European Synchrotron Radiation Facility in Grenoble, France - the technique can now be used on site to map out elements horizontally across the paint surface and vertically through the layers.

The researchers have traced how the composition and thickness of the layers varies from light to shadow on the face of the Mona Lisa, and in the flesh tones of six other paintings by Leonardo in the Louvre. Walter and his team were

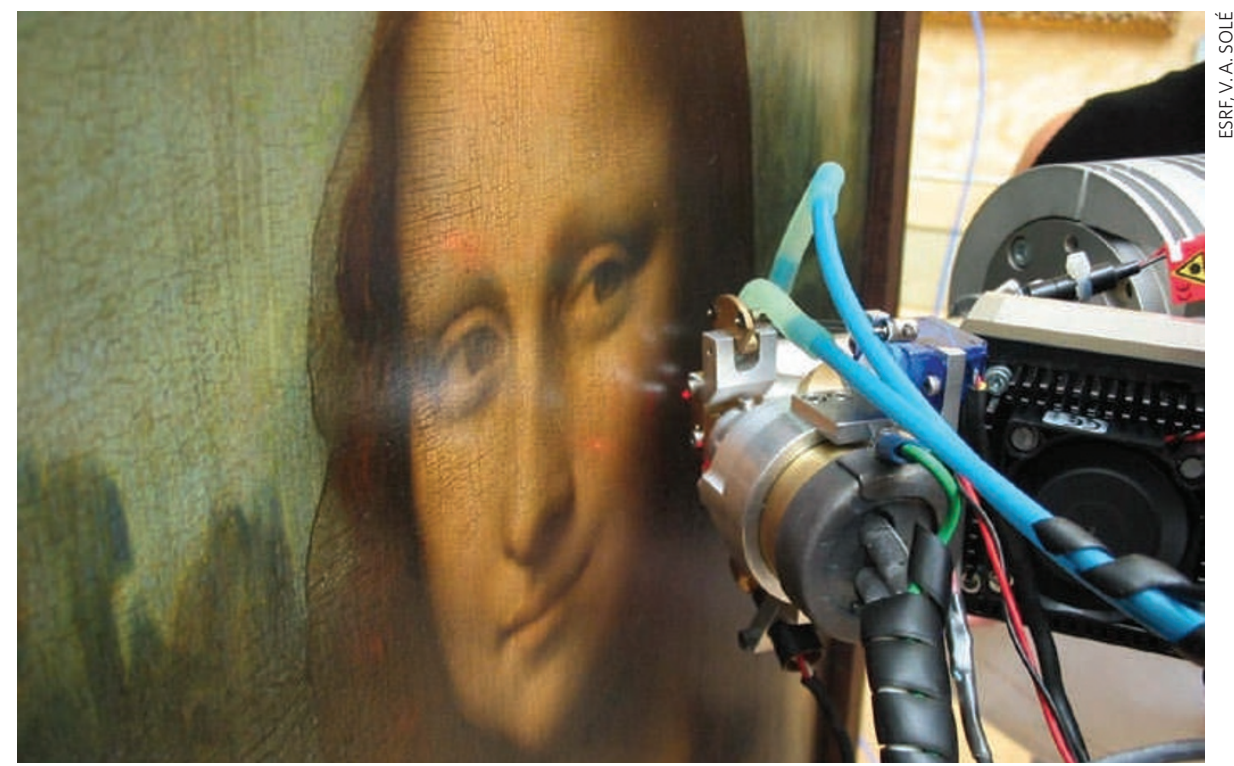

Rare artworks can be analysed on site using X-ray spectroscopy - without removing paint samples.

particularly keen to establish how Leonardo achieved his trademark sfumato ('smoky') shading, which is devoid of evident brush marks.

It was known that this style exploits a glazing technique developed by fifteenth-century northern European oil painters such as Jan van Eyck, in which a translucent paint is laid over an opaque one. But the details of how Leonardo used it to such great effect were obscure. Walter et al. find that the thickness of a brown glaze placed over the pink base of the Mona Lisa's cheek grades smoothly from just 2-5 micrometres to around 30 micrometres in the deepest shadow, and that it is made up of an iron-oxide earth pigment darkened with manganese oxide. Although these materials were widely used, Leonardo's control of glaze thickness is remarkable. He probably used his fingertips, as van Eyck did, rather than a brush.

This finding confirms Leonardo as an innovative artist. He trained in the studio of Andrea del Verrocchio in Florence, but was apparently ready to abandon the pigment-mixing method preferred by Florentine artists in favour of experiments with glazing, similar to those conducted in Venice after the northern European painting techniques reached Italy.

That enthusiasm for experimentation is confirmed by analyses of the other Leonardo paintings, in which he used a variety of materials and techniques, including direct mixing, in the flesh tones. It also tallies with his reputed interest in chemistry, which seemingly provoked Pope Leo's impatience at his tinkering and distilling. It is possible that analyses of other Leonardo paintings might allow his experimental methods to act as chronological markers - a valuable goal, given that the current dating of his works is sketchy.

Prospects for further analyses of the Louvre's paintings on site are challenged by the decision of France's culture ministry to relocate Walter's research laboratories to the town of Neuvillesur-Oise in Cergy-Pontoise, some 30 kilometres outside Paris. The new centre, which is scheduled to operate from 2013, will house conservation laboratories and accommodate 250,000 artworks now stored in the Louvre and other Parisian museums. But the upheaval is taking its toll on staff morale, casting a shadow over the future of scientific research at the Louvre. Philip Ball is a writer based in London. 